\title{
CYR61 as a potential biomarker for the preoperative identification of muscle-invasive bladder cancers
}

\author{
Huang Chen ${ }^{1}$, Yu Liu ${ }^{1}$, Chuanzhen $\mathrm{Cao}^{2}$, Hao Xi ${ }^{1}$, Wenting Chen ${ }^{1}$, Wei Zheng ${ }^{2}$, Xin Dong ${ }^{1}$, Shan Zheng ${ }^{3}$, \\ Lin $\mathrm{Li}^{3}$, Jianhui $\mathrm{Ma}^{2}$, Yanning Gao ${ }^{1}$, Jianzhong Shou ${ }^{2}$ \\ ${ }^{1}$ State Key Laboratory of Molecular Oncology, National Cancer Center/Cancer Hospital, Chinese Academy of Medical Sciences and Peking Union \\ Medical College, Beijing, China; ${ }^{2}$ Department of Urology, National Cancer Center/Cancer Hospital, Chinese Academy of Medical Sciences and \\ Peking Union Medical College, Beijing, China; ${ }^{3}$ Department of Pathology, National Cancer Center/Cancer Hospital, Chinese Academy of Medical \\ Sciences and Peking Union Medical College, Beijing, China \\ Contributions: (I) Conception and design: H Chen; (II) Administrative support: J Ma, Y Gao, J Shou; (III) Provision of study materials or patients: \\ C Cao, H Xi, W Chen, W Zheng, X Dong; (IV) Collection and assembly of data: H Chen, S Zheng, L Li; (V) Data analysis and interpretation: H \\ Chen, Y Liu; (VI) Manuscript writing: All authors; (VII) Final approval of manuscript: All authors. \\ Correspondence to: Yanning Gao; Jianzhong Shou. State Key Laboratory of Molecular Oncology and Department of Urology National Cancer Center, \\ Cancer Hospital, Chinese Academy of Medical Sciences and Peking Union Medical College, 17 Panjiayuan Nanli, Chaoyang District, Beijing \\ 100021, China. Email: yngao@cicams.ac.cn; shoujzh@126.com.
}

Background: The biological behaviors, clinical treatment, prognosis of non-muscle-invasive bladder cancers (NMIBCs) and muscle-invasive bladder cancers (MIBCs) are distinct. Accurate staging is pivotal in optimal therapy planning for bladder cancers (BCs). However, it is insufficient for urologists in preoperative determining whether the tumor has invaded within the muscularis propria through cystoscope and imaging methods (CT or MRI). Therefore, searching for ideal biomarkers from the tumor tissues and urine is important for identifying the MIBCs preoperatively.

Methods: Differentially expressed genes between NMIBCs and MIBCs were identified by microarray analysis and validated by quantitative reverse transcription polymerase chain reaction (qRT-PCR) and immunohistochemical analysis. The correlation between cysteine-rich angiogenic inducer 61 (CYR61) expression and Kaplan-Meier test evaluated patients' overall survival (OS). CYR61 protein levels were measured using enzyme-linked immunosorbent assay (ELISA) in preoperatively collected urine samples from BC patients. The receiver-operating characteristic (ROC) curve analyzed the diagnostic accuracy of uric CYR61. The siRNA mediated silencing of CYR61 in bladder carcinoma cells was performed using Lipofectamine 2000. Cell migration and invasion were assessed using wound healing and transwell assay, respectively.

Results: Differential gene expression analysis using microarray between 14 MIBCs and 16 NMIBCs human tumor samples revealed a significant increase $(\mathrm{P}<0.001)$ in the expression of CYR61 in MIBCs compared with NMIBCs. Higher expression of CYR61 in MIBCs was found in additional 54 tumor samples using qRT-PCR. Therefore, the overexpression of CYR61 in MIBCs could be used as a potential biomarker to distinguish between MIBCs and NMIBCs. ELISA detected elevated levels of CYR61 in the urine samples of MIBC patients (average 2.5-fold) compared with NMIBCs, with $72.7 \%$ sensitivity and $86.0 \%$ specificity to distinguish MIBCs from NMIBCs. Wound healing and transwell assays using CYR61-silenced carcinoma cells indicated the role of CYR61 in cell migration and invasion.

Conclusions: CYR61 expression is higher in MIBCs compared with NMIBCs and can serve as a promising biomarker for the preoperative diagnosis of MIBCs with prognostic value; however, multicentric prospective validation is essential for the further evaluation of CYR61.

Keywords: Bladder cancer (BC); muscle-invasive bladder cancers (MIBCs); non-muscle-invasive bladder cancers (NMIBCs); cysteine-rich angiogenic inducer 61 (CYR61); biomarker 
Submitted Dec 22, 2019. Accepted for publication Feb 28, 2021.

doi: 10.21037/atm-19-4511

View this article at: http://dx.doi.org/10.21037/atm-19-4511

\section{Introduction}

Bladder cancer (BC) is the fourth most common malignancy in males in developed countries and the seventh in China. The incidence of $\mathrm{BC}$ has increased significantly over recent decades $(1,2)$. Approximately $90 \%$ of BCs are urothelium carcinoma, which can be categorized as non-muscleinvasive BCs (NMIBCs) (stages Ta-T1) and muscle-invasive BCs (MIBCs) (stage T2-T4) (3). The biologic behavior, clinical treatment, and prognosis of NMIBCs and MIBCs are distinct. Local recurrence develops in most NMIBCs and can be safely managed by transurethral resection of the tumor (TURBT), while MIBCs might progress into distant metastasis, for which radical cystectomy is regarded as the standard therapy $(4,5)$. In patients initially diagnosed with superficial bladder tumors, upstaging from NMIBC to MIBC was observed in approximately one-third of patients $(6,7)$, and $40 \%$ who underwent TURBT were understaged (8). Therefore, accurate staging is pivotal for optimal therapy planning.

However, preoperative determination or whether the tumor has invaded the muscularis propria is insufficient for urologists using a cystoscope and imaging methods, including computed tomography or magnetic resonance imaging (9-11). There are many recent investigations of the genetic and biologic properties of MIBCs, and researchers are actively searching for ideal bladder tumor markers to identify MIBCs from tissues and urine. Fascin, thrombomodulin, uroplakin (12), stromelysin 3, CD44, E-cadherins, motility-related protein 1, gelsolin, antiadhesive glycoprotein podocalyxin-like, and cathepsin L are potential invasion-related markers found using BC tissue (12-20). Urine- and blood-based markers for MIBCs have been the focus of researchers. Markers, including matrix metalloproteinase (MMP)-2, MMP-9, C-reactive protein, tenascin-C splicing domains, and heparinase (13-16), are expressed relative to MIBCs in blood or urine. However, non-invasive tools that can effectively determine the preoperative staging of BCs remain elusive. Minimally invasive and accurate methods of predicting muscle-invasive urothelial bladder carcinoma are not yet available. Although prior studies have identified several potential serological and urinary markers of MIBCs, to date, no single or set of biomarkers has been systematically verified in rigorously selected sample sets.

The application of high-throughput microarray technology has greatly enabled the search for clinically important disease biomarkers. Several exploratory studies have shown the potential value of gene expression signatures associated with the BC stage (17-20). Our laboratory has also been engaged in screening BC biomarkers since 2001 (21). Therefore, in the present study, we focused on gene expression alterations and analyzed potential biomarkers to distinguish NMIBCs from MIBCs, preoperatively. Consequently, several differential expression genes (DEGs) were identified between NMIBCs and MIBCs. The cysteine-rich angiogenic inducer 61 (CYR61) gene was the top hit, encoding an extracellular matrix protein. CYR61 plays a role in multiple physiological functions, including development, tissue repair, cell adhesion, migration, and proliferation (22). Previous studies have shown CYR61 overexpression is associated with invasion and poor prognosis in several types of cancer, including breast cancer $(23,24)$, gastric cancer $(25,26)$, glioblastoma multiforme $(27,28)$, and oral cancer $(29,30)$. However, the correlation between CYR61 and BC is largely unknown.

In the present study, we focused on CYR61 expression in $\mathrm{BC}$ and explored the possibility of developing it into a biomarker to distinguish MIBCs from NMIBCs.

We present the following article in accordance with the MDAR reporting checklist (available at http://dx.doi. org/10.21037/atm-19-4511).

\section{Methods}

\section{Patients}

The study was conducted in accordance with the Declaration of Helsinki (as revised in 2013). The study was performed at the Cancer Institute (Hospital), Chinese Academy of Medical Science, Beijing, and was approved by the local ethics committee (No. 12-044/578). All patients provided signed informed consent before enrollment.

From June 2012 to March 2015, 84 frozen tissue samples belonging to 84 patients with $\mathrm{BC}$ were obtained from the Department of Urology, Cancer Institute (Hospital), Chinese Academy of Medical Science, Beijing. The 
inclusion criteria were as follows: (I) newly diagnosed BC patients with the largest tumor diameter, $>2.5 \mathrm{~cm}$ and without tumor peduncle, preoperatively; and (II) freshly frozen tissues obtained from $\mathrm{BC}$ patients who underwent radical or partial cystectomy. All samples including in this study are bladder urothelial carcinoma confirmed by surgery and pathology. As transurethral resection of bladder tumor can result in pseudo-lesions and inaccurate staging (31), all fresh frozen tissue including in this study were from BC patients underwent radical or partial cystectomy. For screening, samples from 30 patients were used microarray expression profiling test. Samples from 54 patients were used to validate CYR61 expression by quantitative reverse transcription polymerase chain reaction (qRT-PCR).

Formalin-fixed, paraffin-embedded (FFPE) specimens from 115 patients with bladder urothelium carcinoma underwent surgery at the National Cancer Institute and Hospital, Chinese Academy of Medical Sciences during 1998 and 2010 were selected to to analyze the difference in protein expression by immunohistochemistry (IHC). All 115 patients were confirmed as having a diagnosis of bladder urothelial carcinoma.

For studies on prognostic markers, 104 patients were included between 2012 and 2015. The inclusion criteria were as follows: (I) BC diagnosed by imaging (biopsy); (II) TURBT or radical cystectomy operation was planned; (III) written informed consent was acquired. Urine samples were collected before surgery.

Only one sample per patient was included in the present study. All patients included in the study had a bladder urothelial carcinoma diagnosis, as confirmed by surgery or biopsy.

\section{Data collection}

Baseline data, including sex, age, and clinical information, were collected from all patients enrolled in the study. The tumor stage of all patients was determined according to the 2010 revision of the American Joint Committee on Cancer's Cancer Staging Manual, while tumor grade was determined according to the World Health Organization 2004 grading scheme.

\section{RNA's extraction and microarray expression profiling}

Cystectomy specimens with maximum tumor diameter were immediately reviewed by the pathologist, who visually selected the tumor zone for preservation in RNAlater (Ambion, Austin, TX, USA) at $-80{ }^{\circ} \mathrm{C}$. The remaining cystectomy specimens were fixed in formaldehyde and subjected to standard pathological analysis.

Total RNA was extracted from frozen tissue using TRIzol (Invitrogen, Carlsbad, CA, USA), according to the manufacturer's specifications. The RNA concentration was determined using a NanoDrop ND-1000 Spectrophotometer (NanoDrop Technologies, Wilmington, DE, USA). RNA's integrity was determined using a 2100 Bioanalyzer (Agilent Technologies, Santa Clara, CA, USA). The total RNA used in the present study had an optical density of 260/280 ratios $>1.9$ and RNA integrity number $>7.0$.

The labeling, hybridization, washing, and scanning of samples was conducted according to the manufacturer's instructions. Briefly, $1.65 \mu \mathrm{g}$ of Cy3-labeled cRNA was generated from $500 \mathrm{ng}$ of total RNA by in vitro transcription using a Low RNA Input Linear Amplification Kit PLUS (Agilent, USA) and hybridized to the Agilent $4 \times 44 \mathrm{~K}$ Whole Human Genome Oligo Microarrays (G4112F; Agilent, USA). After hybridization, the slides were washed and scanned with the Agilent G2505B Microarray Scanner System (Agilent, USA). Fluorescence intensities on scanned images were extracted and preprocessed using Agilent Feature Extraction Software (version 9.1; Agilent, USA). Raw data were normalized by the median scale method using the R package limma (www.r-project.org). Microarray data were deposited in the NCBI Gene Expression Omnibus (series accession no. GSE77952).

\section{$q R T-P C R$}

Primer pairs were designed to span at least 1 intron to avoid amplifying the contaminating genomic DNA and cDNA. Primer pair of CYR61 (forward: AT C C C T G GAT T GAA G C GCAA, revers e : CACTGCAACGTCAAGGTTCG) amplified a product of 137 bp' $1 \mu \mathrm{g}$ total RNA was reverse transcribed using random primers (Promega, Madison, WI, USA) in a reaction volume of $20 \mu \mathrm{L}$ using SuperScript II Reverse Transcriptase (Invitrogen, Carlsbad, CA, USA) according to the manufacturer's instructions. qRT-PCR was performed using SYBR Premix Ex Taq (TaKaRa, Dalian, China) and Mx3005p thermal cycler (Stratagene, La Jolla, CA, USA), according to the manufacturers' recommendations, in a $25 \mu \mathrm{L}$ final reaction volume. Cycle thresholds $(\mathrm{Ct})$ were calculated for each reaction, and $18 \mathrm{~S}$ was used as the reference gene. All samples were analyzed in triplicate, and the Ct mean 
calculated for data analysis using the comparative $\mathrm{Ct}$ method for relative gene expression, as previously described (32).

\section{IHC}

CYR61 protein expression in tumor cells was further quantified, as described previously (25). Protein expression patterns of CYR61, whose mRNA expression was significantly higher in MIBCs, identified by the mRNA microarray and qRT-PCR analyses, were assessed in

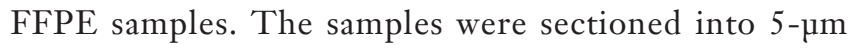
slices and mounted on glass slides. CYR61 staining was performed following heat-based antigen retrieval using a mouse monoclonal antibody against CYR61 (ab80112; dilution 1:400; Abcam, Cambridge, UK). All slides were independently scored by two pathologists, who were blinded to the stage and outcome. The results of the CYR61 IHC were scored on a scale of $0-3$, where level 0 was negative or $<5 \%$ tumor cells stained, intensity level 1 was $5-50 \%$ tumor cells stained, intensity level 2 was $50-80 \%$ of tumor cells stained, and intensity level 3 was $>80 \%$ tumor cells stained. A high expression was scored as $2-3$. The association of CYR61 with time to progression and overall survival (OS) was evaluated at the protein level using a subset of 108 cases with available follow-up data. In this subset of 108 patients with BC, BC's recurrence or progression had to be proven by cytological biopsy or surgery, and 16 cases died of BC. The time to recurrence or OS was recorded as time from surgery to the relevant event. Survival data were updated on June 1st, 2014.

\section{Urine}

According to the standard protocol for urine collection developed by the Human Urine and Kidney Proteome Project and European Kidney and Urine Proteomics EuroKUP Initiatives (www.eurokup.org; www.hukpp.org), $>50 \mathrm{~mL}$ mid-stream morning second urine samples were sent to the laboratory within $30 \mathrm{~min}$ and centrifuged at $1,000 \times \mathrm{g}$ for $10 \mathrm{~min}$. The resulting supernatant was divided into several 1 - and $10-\mathrm{mL}$ aliquots and frozen at $-80^{\circ} \mathrm{C}$. All urine samples were freeze-thawed only once.

Levels of human CYR61 (DY4055; R\&D Systems, MN, USA), Vascular Endothelial Growth Factor C (VEGFC; DVEC00, R\&D Systems, MN, USA), vascular endothelial growth factor (VEGF) (DY293B; R\&D Systems, MN, USA), and MMP-2 (DY902, R\&D Systems, MN, USA) were tested in urine samples using commercial enzyme- linked immunosorbent assay (ELISA) according to the manufacturer's instructions. According to the manufacturer's instructions, calibration curves were prepared using purified standards for each assessed protein and fitted by linear logistic regression. Absorbance values were read on a microplate reader (Synergy HT; BioTek, Winooski, VT, USA) at a wavelength of $450 \mathrm{~nm}$. Every sample was analyzed in triplicate, and the mean was calculated for data analysis. Due to the unavoidable variability of voided urine concerning total volume and time in the bladder, each biomarker was normalized to urine creatinine (KGE005; R\&D Systems, MN, USA), as previously described (33).

\section{Cell lines}

Bladder carcinoma cell lines T24, J82, UMUC-3, and 5637, and immortalized human urothelial SV-HUC-1 cells, were obtained from the Cell Resource Center of the Institute of Basic Medical Sciences, Peking Union Medical College (Beijing, China). Cells were cultured in RPMI 1640 supplemented with $10 \%$ at $37{ }^{\circ} \mathrm{C}$ in a humidified atmosphere of $97.5 \%$ air and $5 \% \mathrm{CO}_{2}$.

\section{Transfection}

CYR61 transiently silenced cell lines were generated by transfecting CRY61 siRNA using Lipofectamine 2000 (Invitrogen, Carlsbad, CA, USA) following the manufacturer's protocol. After $24 \mathrm{~h}$, cells were harvested for western blot and qRT-PCR assays.

\section{Antibodies used for western blot}

The following antibodies were used for western blot: CYR61 (ab80112; dilution 1:1,000; Abcam, UK) and $\beta$-actin (sc-47778; dilution 1:500; Santa Cruz Biotech, Dallas, TX, USA).

\section{Cell migration}

Scratch assays or wound closure assays have been routinely used to investigate cell migration. Briefly, $25 \times 10^{4}$ cells $/ \mathrm{mL}$ were seeded onto a $60 \mathrm{~mm}$ cell culture dish and incubated for $24 \mathrm{~h}$ to obtain a $100 \%$ confluency. A scratch was made using a p200 pipette to create the wound. Cell debris was removed by washing 3 times with phosphate-buffered saline. The size of the wounds was similar in the CYR61 transiently silenced cell lines and the control. Cells were 
further incubated for $24 \mathrm{~h}$ in a fresh culture medium. Cell migration was assessed from the gap closure, as analyzed from photographs, using Image J software (version 1.46; National Institutes of Health, Bethesda, MA, USA).

\section{Cell invasion}

Cell invasion was analyzed using a transwell chamber (24 wells, 8.0- $\mu$ m pore size; Corning, NY, USA). Cells cultured on the transwell chamber were subjected to serum deprivation for $24 \mathrm{~h}$. The transwell chamber was then transferred to a 6-well plate with complete media. Cells were allowed to migrate for $24 \mathrm{~h}$; subsequently, cells on top of the filter were removed using a cotton swab and the cells were fixed in formalin for 10 min before staining with $0.1 \%$ crystal violet for $30 \mathrm{~min}$. Cells were fixed and stained with crystal violet $(0.1 \%$; lower). The number of migrated cells was counted using an inverted microscope.

\section{Statistical analysis}

mRNA microarray raw data with global median were normalized with GeneSpring GX software, version 11.5 (Silicon Genetics, Redwood City, CA, USA) and analyzed using $\mathrm{R}$ statistical software, version 2.8.1. The MannWhitney $\mathrm{U}$-test assessed the differences in gene expression values and urinary protein concentrations between groups. The association of CYR61 expression with histopathological stage and other parameters was evaluated using $\chi^{2}$-test or Student's $t$-test. The association of CYR61 expression levels with progression-free survival (PFS) and OS was analyzed using the Kaplan-Meier test, and Kaplan-Meier curves were compared by the log-rank test. The receiver-operating characteristic (ROC) curve was used to calculate the area under the curve (AUC) and the 95\% confidence interval (CI) associated with MIBCs and NMIBCs. AUC curves were plotted, and 95\% CIs were calculated in GraphPad Prism 5.0 (GraphPad Software Inc., San Diego CA, USA). Statistical analyses were performed using the SPSS statistical package, version 17.0 (SPSS Inc., Chicago, IL, USA). $\mathrm{P}<0.05$ indicated statistical significance.

\section{Results}

\section{Baseline characteristics of patients enrolled in the study}

To identify and establish the diagnostic value of the biomarkers that can distinguish MIBCs from NMIBCs, patients age $\geq 60$ years were divided into four groups as follows: screening set $(\mathrm{n}=30)$, validation set $(\mathrm{n}=54)$, urine set $(\mathrm{n}=104)$, and FFPE set $(\mathrm{n}=115)$ (Table 1 and Table S1). No significant difference $(\mathrm{P}>0.05)$ was detected between groups concerning sex, alcohol consumption, smoking history, tumor grade, multifocality, and recurrence.

\section{CYR61 overexpression in MIBCs compared with NMIBCs}

To identify the biomarker that could distinguish MICBCs from NMIBCs, 30 human bladder tumors, including 14 MIBCs and 16 NMIBCs, were subjected to transcriptome analysis by microarray (Figure 1A). CYR61 transcript level was significantly $(\mathrm{P}<0.001)$ higher in MIBCs than in NMIBCs, with a 3.34-fold change.

Data from the microarray analysis were further verified by qRT-PCR of the samples used in the microarray. The expression of CYR61 transcripts was significantly higher (3.56-fold, $\mathrm{P}=0.005)$ in MIBCs compared with NMIBCs (Figure 1B).

To validate the role of CYR61 as a potential biomarker to distinguish between MIBC- and NMIBC-independent cohorts, 54 tumor samples (22 MIBCs and 32 NMIBCs) were analyzed by qRT-PCR. Significantly greater (2.17-fold change, $\mathrm{P}=0.016)$ CYR61 transcript levels were observed in MIBCs compared with NMIBCs (Figure 1C).

The results indicated that CYR61 was overexpressed in MIBCs compared with NMIBCs.

\section{CYR61 protein overexpression in MIBCs and its association with poor prognosis}

We observed a high CYR61 expression (defined as a score of $2-3)$ in urothelial tumor cells $(40.8 \%, 47 / 115)$, but not in normal urothelium cells $(3.5 \%, 4 / 115)$ (Figure $2 A)$. The results indicated a potential role of CYR61 in BC. The role of CYR61 as a prognostic marker was further evaluated by IHC of 115 FFPE bladder tumors, which included 59 NMIBCs (5 Ta, $54 \mathrm{~T} 1$ ) and 56 MIBCs (37 T2, 16 T3, 3 T4).

Furthermore, CYR61 expression was significantly associated with tumor stage; high CYR61 expression was detected in $64.9 \%(37 / 57)$ MIBCs compared with $17.2 \%(10 / 58)$ NMIBCs $\left(\chi^{2}\right.$-test, $\left.\mathrm{P}<0.001\right)$ (Table 2 and Figure $2 A)$. No significant correlation was observed between high CYR61 expression and other clinicopathological parameters.

Survival analysis was carried out among 108 patients with the available follow-up information (median follow-up 
Table 1 Clinical and pathological characteristics of patients included in the present study

\begin{tabular}{|c|c|c|c|c|}
\hline Characteristics & Screening set $(n=30)$ & Validation set $(n=54)$ & FFPE set $(n=115)$ & Urine set $(n=104)$ \\
\hline Mean \pm SD & $63.62 \pm 12.73$ & $62.28 \pm 11.35$ & $60.81 \pm 11.47$ & $63.99 \pm 10.15$ \\
\hline Median [range] & $66.0[34-78]$ & $64.5[33-84]$ & $62.0[32-82]$ & $64.0[30-84]$ \\
\hline \multicolumn{5}{|l|}{ Sex } \\
\hline Female & $12(40.0)$ & $7(12.96)$ & $25(21.7)$ & 19 (19.3) \\
\hline \multicolumn{5}{|c|}{ Alcohol consumption } \\
\hline Yes & $7(23.3)$ & $12(22.2)$ & $14(12.2)$ & $20(23.8)$ \\
\hline No & $23(76.7)$ & $42(77.8)$ & $101(87.8)$ & $84(76.1)$ \\
\hline No & $20(66.7)$ & $32(59.3)$ & $57(49.6)$ & $63(60.6)$ \\
\hline \multicolumn{5}{|l|}{ Tumor stage } \\
\hline Non-invasive & $16(50.0)$ & $32(59.26)$ & $59(51.3)$ & $81(77.9)$ \\
\hline $\mathrm{Ta}$ & 2 & 6 & 5 & 30 \\
\hline T1 & 14 & 26 & 54 & 51 \\
\hline Invasive & $14(50.0)$ & $22(40.74)$ & $56(48.7)$ & $23(22.1)$ \\
\hline $\mathrm{T} 2$ & 11 & 12 & 37 & 13 \\
\hline T3 & 3 & 9 & 16 & 9 \\
\hline \multicolumn{5}{|l|}{ Tumor grade } \\
\hline Yes & $18(60.0)$ & $33(61.1)$ & $43(37.4)$ & $47(45.2)$ \\
\hline No & $12(40.0)$ & $21(38.9)$ & $72(62.6)$ & 57 (54.8) \\
\hline
\end{tabular}

FFPE, formalin-fixed, paraffin-embedded; SD, standard deviation.

interval of 53 months, range: 2-120 months). Furthermore, we found that the expression patterns of CYR61 in BC were significantly associated with patients' $\mathrm{OS}(\mathrm{P}=0.041)$ (Figure 2B). Patients with high CYR61 expression's median survival was 94 months (95\% CI: 79.1-110.0) compared with 110.2 months (95\% CI: 103.7-116.7) in patients with low CYR61 expression. The 5-year survival rate of patients with high CYR61 expression was 70\%, while patients with low CYR61 expression were 91\% (Figure 2C). Further analysis found that CYR61 expression had an independent impact on the OS of NMIBCs and MIBCs (Figure S1). However, no significant association was detected between CYR61 expression and disease-free survival rate in the present study.

\section{CYR61 as a diagnostic marker to distinguish MIBCs from NMIBC}

Mature CYR61 protein is secreted into the extracellular region and can be detected in patients' urine samples. 
A

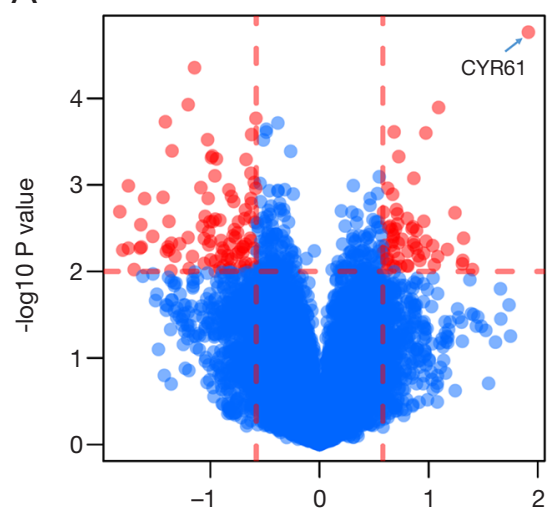

B

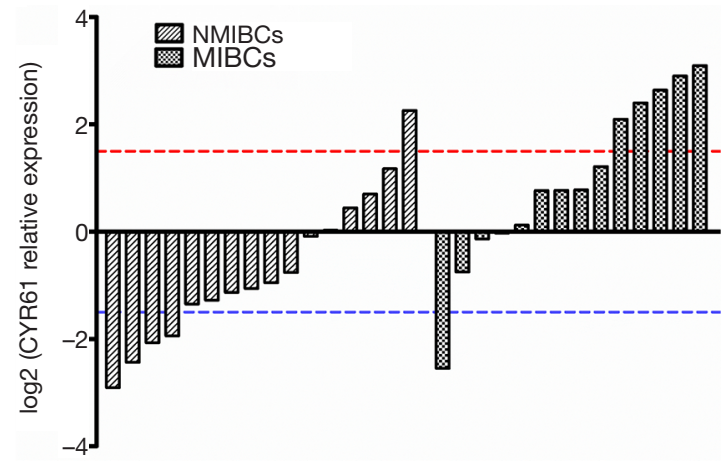

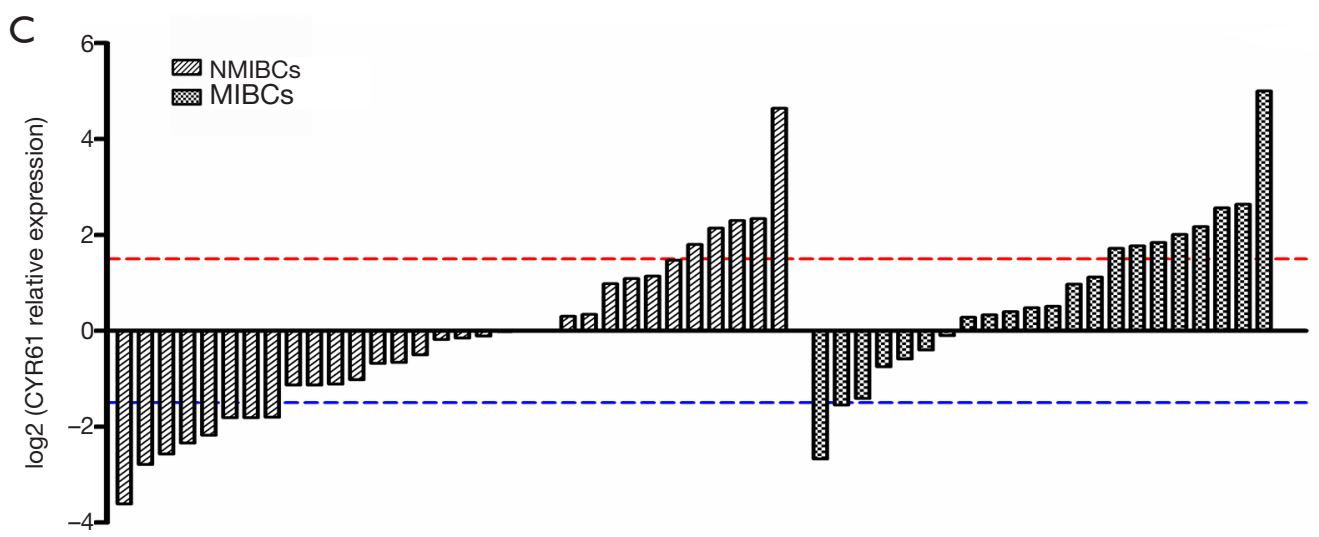

Figure 1 Significant difference of CYR61 expression between NMIBCs and MIBCs. (A) Volcano plot shows different gene expressions between NMIBCs and MIBCs. Two vertical lines represent the 1.5 -fold change boundaries, and the horizontal line is the statistical significance boundary $(\mathrm{P}<0.01)$. Genes with $\geq 2$-fold change and statistical significance are marked with red dots. (B) First phase of validation and relative expression level of CYR61 of screening set (30 samples) tested by qRT-PCR. (C) Second phase of validation. Relative expression level of CYR61 in 32 NMIBCs and 22 MIBCs of independent set tested by qRT-PCR. CYR61, cysteine-rich angiogenic inducer 61; NMIBC, non-muscle-invasive bladder cancer; MIBC, muscle-invasive bladder cancer; qRT-PCR, quantitative reverse transcription polymerase chain reaction.

ELISA analyzed the diagnostic value of CYR61 and the distinction between MIBCs and NMIBCs preoperatively in the urine samples of 104 bladder patients (72 patients who underwent TURBT and 32 patients who underwent cystectomy). A total of 81 bladder tumors were pathologically diagnosed as NMIBCs (51 T1, 30 Ta) and 23 as MIBCs (T2-4) following surgery (Table 1). The urinary CYR61 expression was determined by ELISA and normalized to urinary creatinine levels. Initially, we found a 2.5 -fold higher CYR61 concentration in MIBC patients $(10.48 \pm 9.36 \mathrm{ng} / \mathrm{mg})$ compared with NMIBC patients $(4.12 \pm 2.26 \mathrm{ng} / \mathrm{mg}, \mathrm{P}<0.001)$ (Figure $3 A$ ), which was consistent with the findings at the transcription level and in protein translation in tumor tissues. Next, we explored the possibility of using urinary CYR61 level as a biomarker to distinguish MIBCs from NMIBCs in the ROC curve. The AUC curve was 0.883 (95\% CI: 0.804-0.962) for MIBCs vs. NMIBCs $(\mathrm{P}<0.001)$, with $72.7 \%$ sensitivity and $86.0 \%$ specificity (Figure 3B). Therefore, the detection of CYR61 in urine could serve as a potential biomarker for differentiation between MIBCs and NMBICs.

\section{CYR61 plays a role in the migration and invasion of invasive bladder carcinoma cells}

Endogenous levels of CYR61 were higher in MIBCs (T24, J82, and UMUC-3) compared with NMBICs (5637) (Figure S2). To assess the role of CYR61 in cell migration 

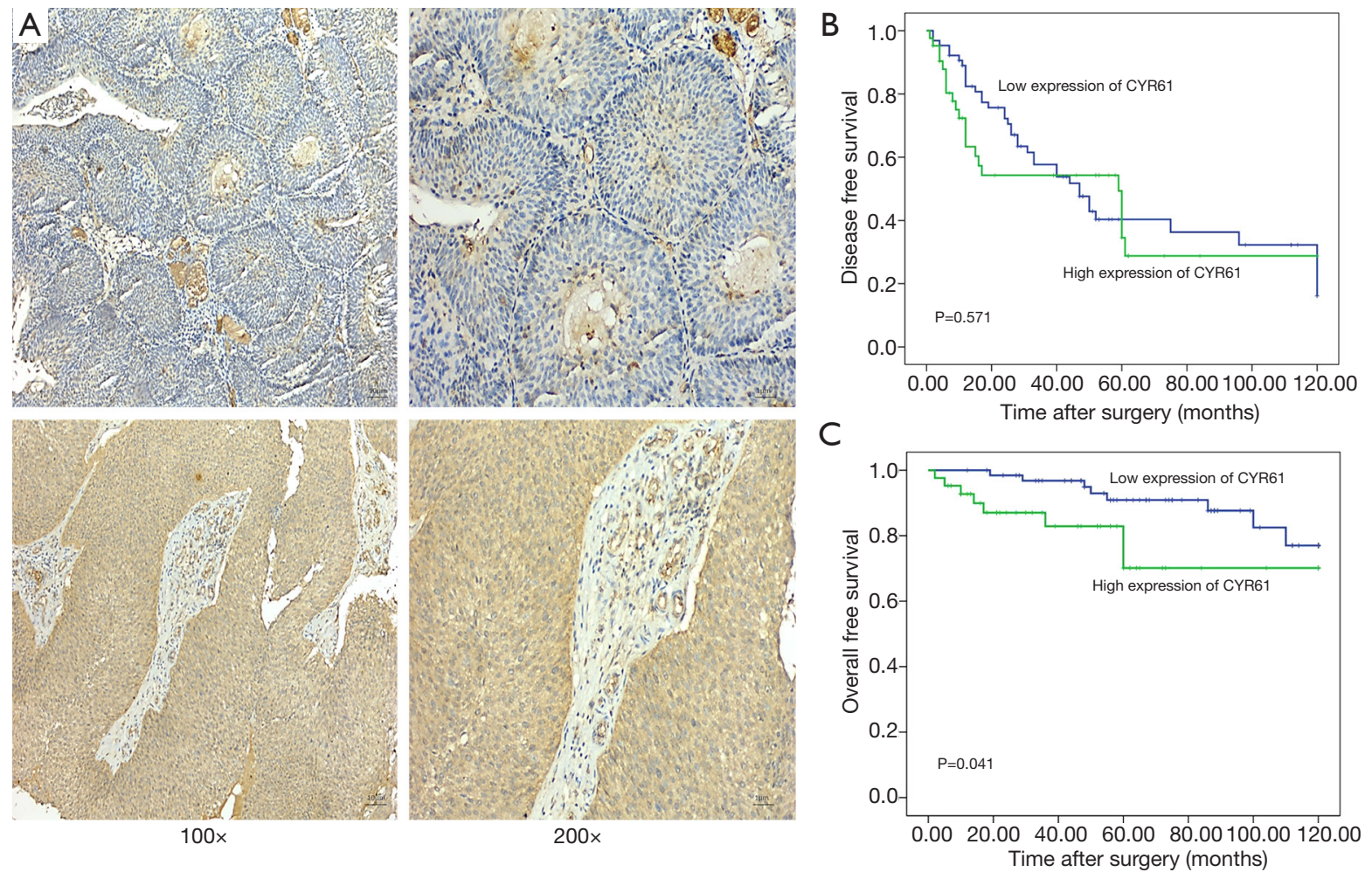

Figure 2 Representative CYR61 IHC of BC. (A) Low CYR61 expression is shown in NMIBCs (top left 100×, top right 200×, Ta stage); high CYR61 protein expression in MIBCs (lower left 100×, lower right 200×, T2 stage). (B) Kaplan-Meier survival analysis of CYR61 expression in 108 BC patients (log-rank test). Relationship of CYR61 expression and patients' OS: low expression, $n=66$; high expression, $\mathrm{n}=42$ ( $\mathrm{P}=0.041$, log-rank =4.191). (C) Disease-free survival curves from the time of BC diagnosis by CYR61 expression. Low expression, $\mathrm{n}=66$; high expression, $\mathrm{n}=42(\mathrm{P}=0.571$, log-rank =0.312). CYR61, cysteine-rich angiogenic inducer 61; IHC, immunohistochemistry; BC, bladder cancer; NMIBC, non-muscle-invasive BC; MIBC, muscle-invasive BC; OS, overall survival.

and invasion, scratch wounding and transwell chamber migration assays were performed. As in the gene expression profiling of 30 human bladder tumors, we finished, the expression of CYR61 demonstrated a significant correlation with MMP-2 and neuropilin-1 (NRP-1) expression (Figure S4). The effect of CYR61 on MMP-2 and NRP1 expression was also observed in vitro. The overexpression of CYR61 in 5637 showed no effect in increasing wound healing, transwell chamber migration, and MMP-2 and NRP-1 expression $(\mathrm{P}>0.05)$ (Figure 4$)$, but migration and invasion in UMUC-3 increased, as well as the expression of MMP-2 and NRP-1 ( $\mathrm{P}<0.05)$ (Figure 5). As expected, scratch wounding and transwell chamber migration assays exhibited that the transiently silence of CYR61 inhibited MIBC cells migration (T24, scratch wounding assay: $\mathrm{P}<0.05$, transwell chamber migration assay: $\mathrm{P}<0.05$; J82, scratch wounding assay: $\mathrm{P}<0.05$, transwell chamber migration assay: $\mathrm{P}<0.05$ ) (Figure 6). MMP-2 and NRP-1 expression decreased at the same time $(\mathrm{P}<0.05)$ (Figure 6).

\section{Discussion}

In the present study, we found that MIBCs express significantly higher levels of CYR61 compared with NMIBCs. Expression levels of CYR61 in MIBCs are associated with tumor stage prognosis. Urinary levels of CYR61 could be used as a biomarker to assess MIBCs. Our findings confirmed that the expression of CYR61 is crucial for the migration and invasion potential of invasive $\mathrm{BC}$ cell lines.

A high-throughput microarray screening of mRNA expression in 30 bladder tumors was performed, followed by DEGs screening between NMIBCs and MIBCs. Subsequently, another set of 56 bladder tumor samples was validated, and the candidate genes were verified by qRTPCR. CYR61, encoding a secreted protein, is the most 
Table 2 Clinical and pathological characteristics for CYR61 expression in FFPE samples (115 bladder urothelium carcinoma)

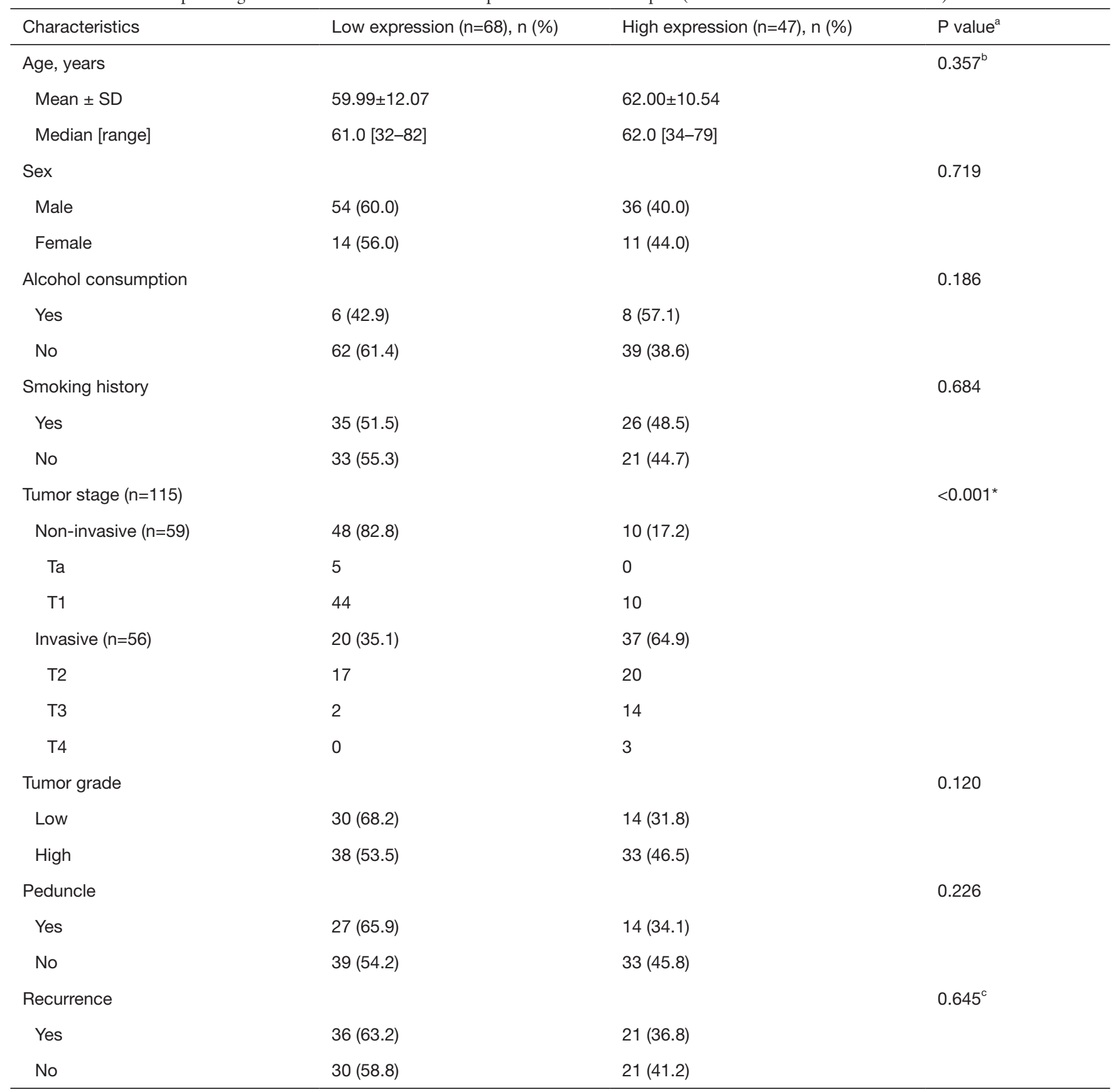

*, $\mathrm{P}<0.05$ indicated statistical significance; ${ }^{\mathrm{a}}, \chi^{2}$-test; ${ }^{\mathrm{b}}$, Student's $t$-test; ${ }^{\mathrm{c}}, 7$ of 115 patients were lost to follow-up. CYR61, cysteine-rich angiogenic inducer 61; FFPE, formalin-fixed, paraffin-embedded; SD, standard deviation.

significant DEG in NMIBCs and MIBCs, assessed by IHC, and was further detected in urine samples by ELISA.

In the present study, all freshly frozen BC tissue samples in the screening and independent sets were obtained from BC patients who underwent radical or partial cystectomy to ensure accurate staging of NMIBCs and MIBCs. The $\mathrm{NMIBC}$ and MIBC groups were subjected to a rigorous selection and were matched in age, sex, and tumor characteristics using a single-variable method. However, we pursued the biomarkers based on the microarray 

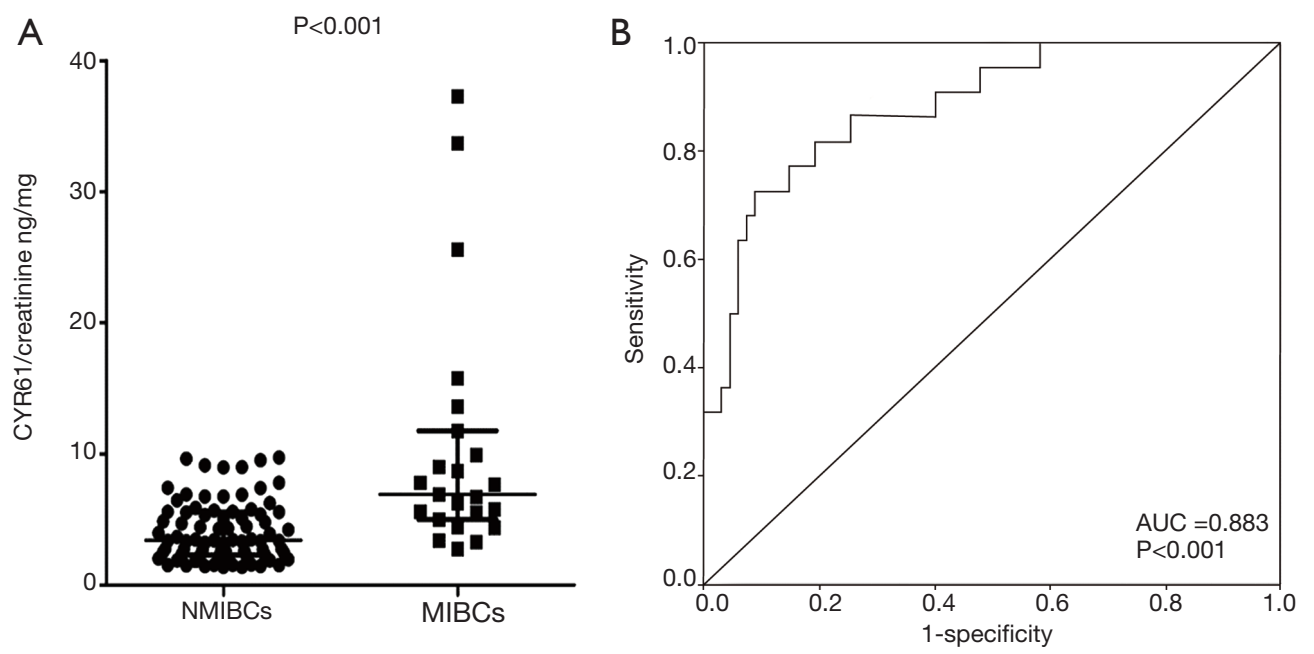

Figure 3 The diagnostic value of CYR61 and the distinction between MIBCs and NMIBCs preoperatively in the urine samples. (A) Urine concentration of CYR61 in NMIBCs and MIBCs. Significance was assessed by Wilcoxon rank sum test $(\mathrm{P}<0.001)$. (B) ROC curves of CYR61 in discriminating between NMIBCs and MIBCs; AUC was 0.883 (95\% CI: 0.804-0.962) for MIBCs vs. NMIBCs (P<0.001), with $72.7 \%$ sensitivity and $86.0 \%$ specificity. CYR61, cysteine-rich angiogenic inducer 61; NMIBC, non-muscle-invasive bladder cancer; MIBC, muscle-invasive bladder cancer; ROC, receiver-operating characteristic; AUC, area under the curve; CI, confidence interval.

expression profile analysis of NMIBCs and MIBCs and performed 2-phase qRT-PCR verification. Subsequently, the most obvious DEG with maximum invasion potential related to CYR61 was selected, and the variable protein expression in NMIBCs and MIBCs was verified. CYR61 is a secreted protein that was further tested in the urine. MMP-2, VEGFC, and VEGF demonstrated differential expression in NMIBCs and MIBCs and demonstrated a positive association with CYR61 in our microarray expression profiling (data not shown). A significant increase in the urinary CYR61 level in MIBCs was observed compared with NMIBCs; urinary CYR61 was significantly higher in $\mathrm{BC}$ patients than controls without $\mathrm{BC}(\mathrm{P}=0.007)$ (Figure S3).

The prognostic value of CYR61 overexpression in tissue has been previously studied in breast cancer $(23,24)$, gastric cancer $(25,26)$, glioblastoma multiforme $(27,28)$, oral cancer $(29,30)$, and ovarian epithelial carcinoma (34). However, as a soluble diagnostic or prognostic biomarker, CYR61 has been only studied in acute coronary syndrome and systemic lupus erythematosus (35). Serum samples were examined for CYR61 level in Klingenberg et al. and Lin et al.'s studies $(35,36)$. To the best of our knowledge, the present study is the first to analyze the urinary level of CYR61 in BC. CYR61 is an extracellular matrix protein with multiple physiological functions, including development, tissue repair, cell adhesion, migration, and proliferation (22). In the present study, we evaluated the diagnostic performance of CYR61, MMP-2, VEGFC, and VEGF to distinguish MIBCs from NMIBCs. CYR61 levels $(\mathrm{AUC}=0.883, \mathrm{P}<0.001)$ demonstrated high sensitivity $(72.7 \%)$ and specificity (86.0\%) to distinguish MIBCs from NMIBCs compared with other markers (Figure 3B, Figure S3, Table S2). Schiffer et al. established and validated a panel of 4 urinary polypeptides uromodulin, collagen $\alpha-1$ (I), collagen $\alpha-1$ (III), and a membrane-associated progesterone receptor component that seemed promising for the non-invasive detection of MIBCs, and the panel revealed a sensitivity of $81 \%$ (95\% CI: $69-90 \%)$ and specificity of $57 \%$ (95\% CI: $45-69 \%$ ) for muscle-invasive disease (37). Compared with previous study, CYR61 showed similar sensitivity and higher specificity in our study. Also, we found that CYR61 expression levels were significantly related to tumor stages. The findings revealed CYR61 to be an efficient marker for poor prognosis. This is the first study to report on CYR61 expression in the tissue and urine of patients with bladder urothelium carcinoma and find that CYR61 is correlated with clinical stages and prognosis of BC. Based on our findings, CYR61 could be used as a diagnostic marker to differentiate MIBCs from NMIBCs. CYR61 expression levels could also potentially serve as a marker for poor prognosis. 
A
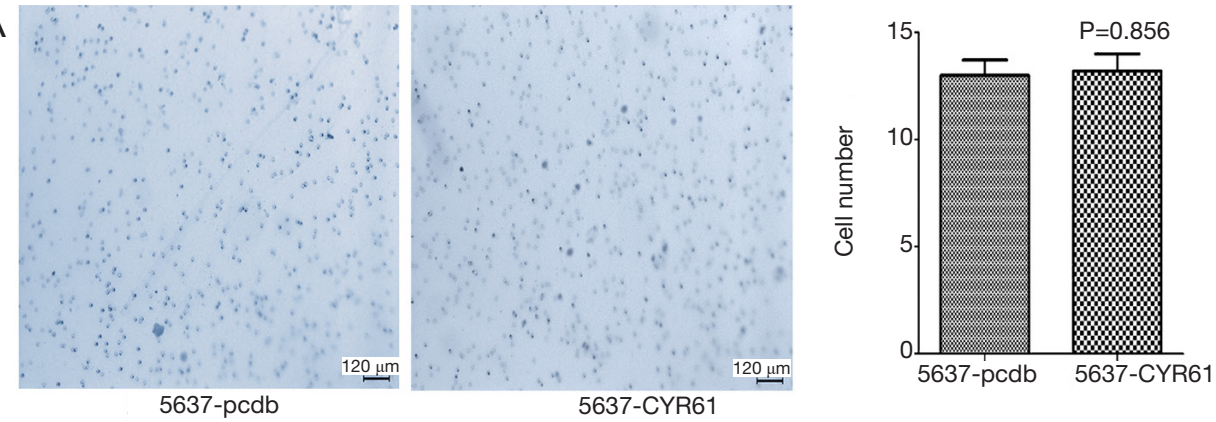

B
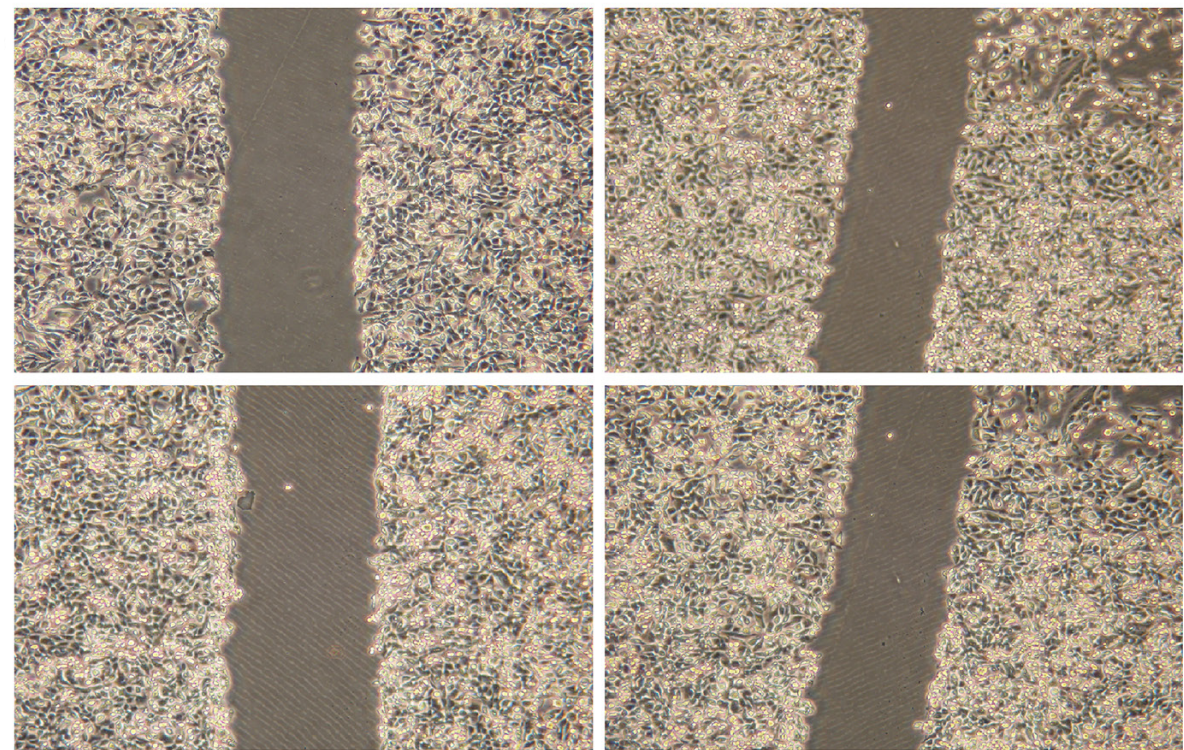

$5637-p c d b$

C

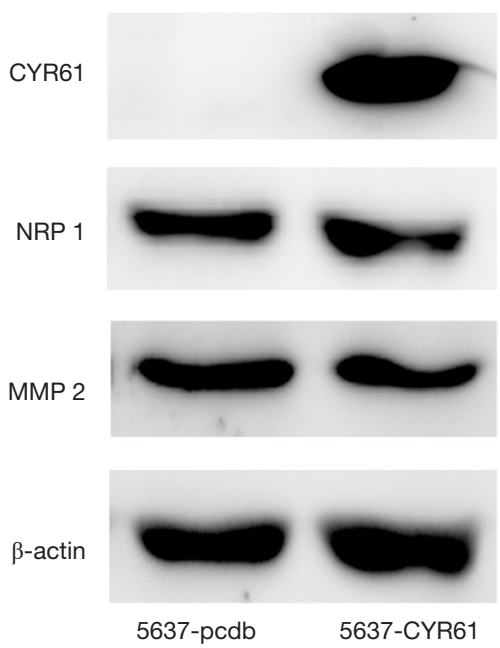

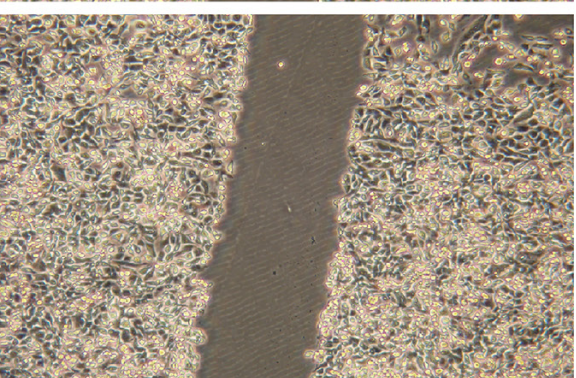

5637-CYR61

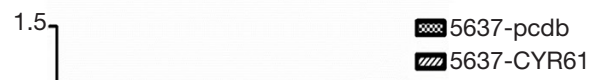

5637-CYR61

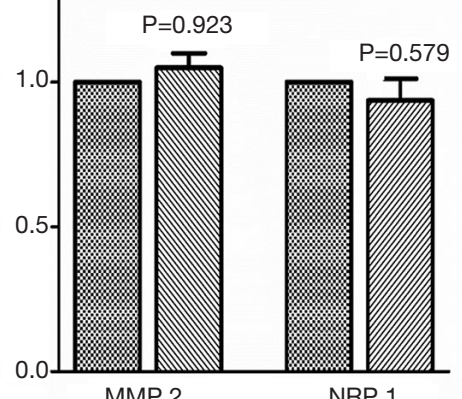

MMP 2

NRP 1

Figure 4 Overexpressed CYR61 cannot increase migration and invasion in 5637 cells. Compared with blank control group (5637-pcdb), no difference was observed between overexpressed CYR61 group (5637-CYR61). (A) Migration through transwell chamber migration assay $(\mathrm{P}<0.05)$; scale bars: $120 \mu \mathrm{m}$. (B) Wound closure in scratch wounding migration assay $(100 \times)$. Photographs were taken at 36 h. $(\mathrm{C})$ Expression of MMP-2 ( $\mathrm{P}=0.923)$ and NRP-1 (P=0.579). CYR61, cysteine-rich angiogenic inducer 61; MMP-2, matrix metalloproteinase-2; NRP-1, neuropilin-1. 
A
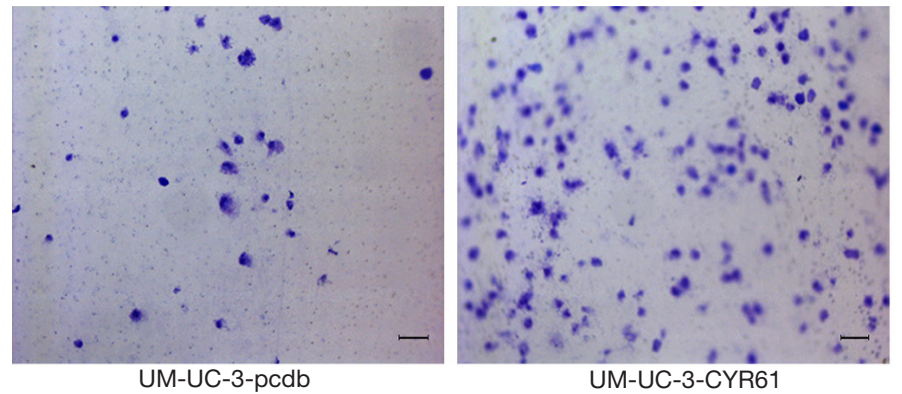

B
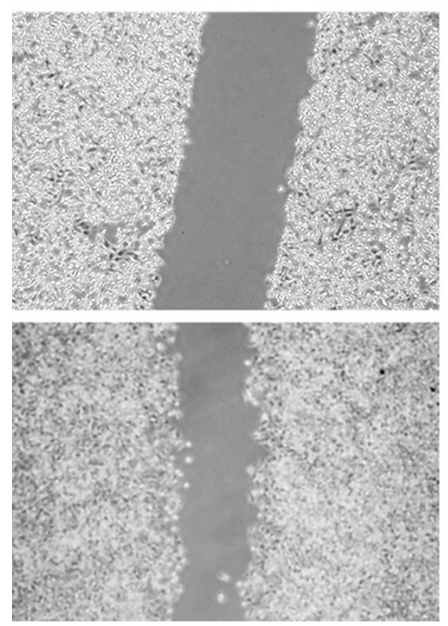

UM-UC-3-pcdb

C

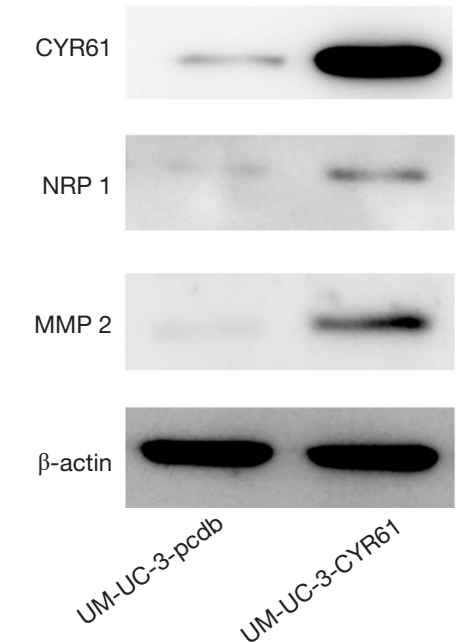

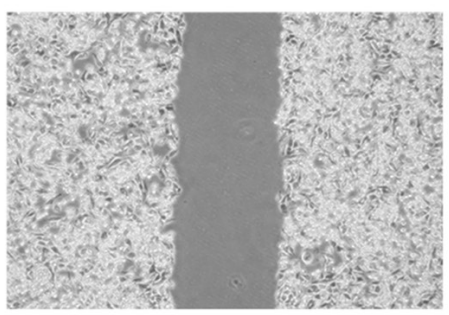

$\mathrm{Oh}$

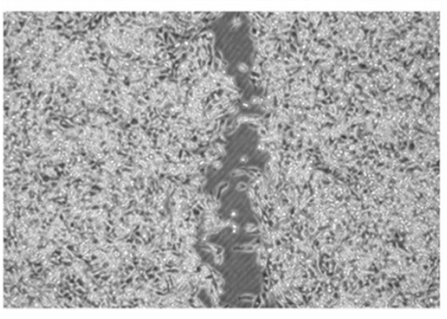

UM-UC-3-CYR61

$24 \mathrm{~h}$

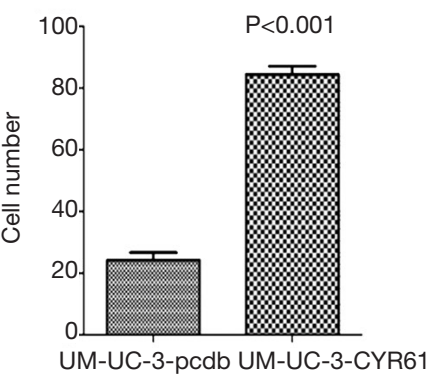

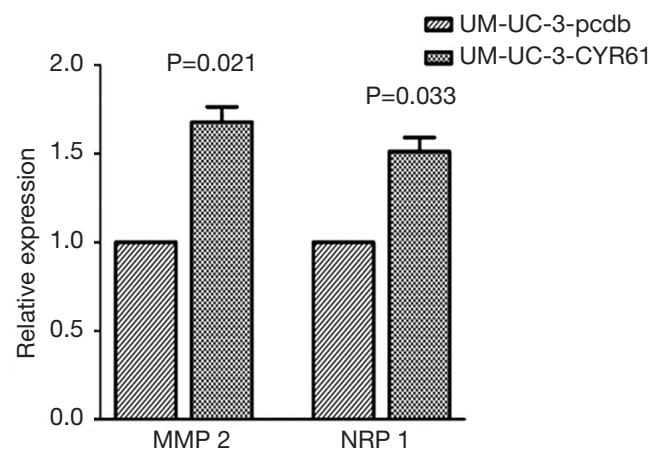

Figure 5 Overexpressed CYR61 increased migration and invasion in UMUC-3 cells. UM-UC-3 -pcdb: UM-UC-3 cells transfected with the pcDNA3.1 blank plasmid server as blank control group; UM-UC-3-CYR61: over-expression of CYR61 in UMUC-3 cells. (A) Migration though transwell chamber migration assay $(\mathrm{P}<0.001)$, scale bars: $120 \mu \mathrm{m}$. (B) Wound closure in scratch wounding migration assay $(100 \times)$. Photographs were taken at $24 \mathrm{~h}$. (C) Upregulated expression of MMP-2 ( $\mathrm{P}=0.021)$ and NRP-1 (P=0.033). CYR61, cysteine-rich angiogenic inducer 61; MMP-2, matrix metalloproteinase-2; NRP-1, neuropilin-1. 
A
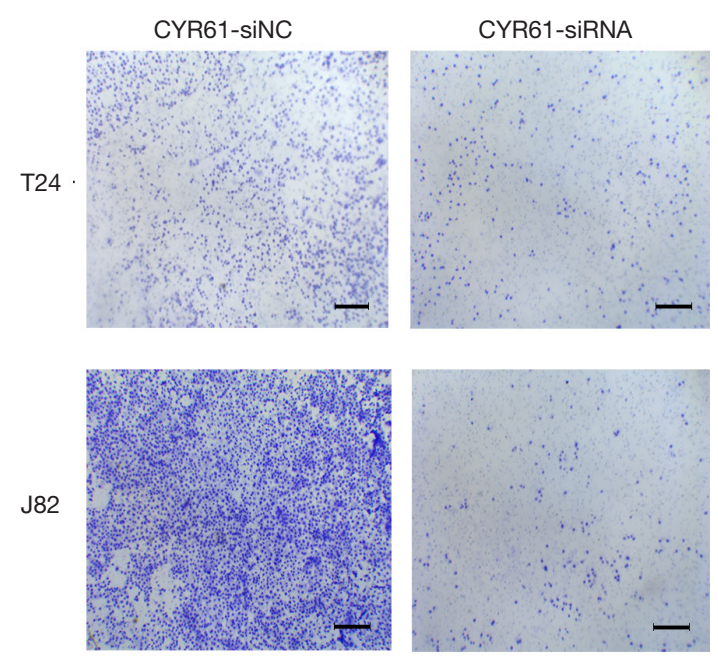

B
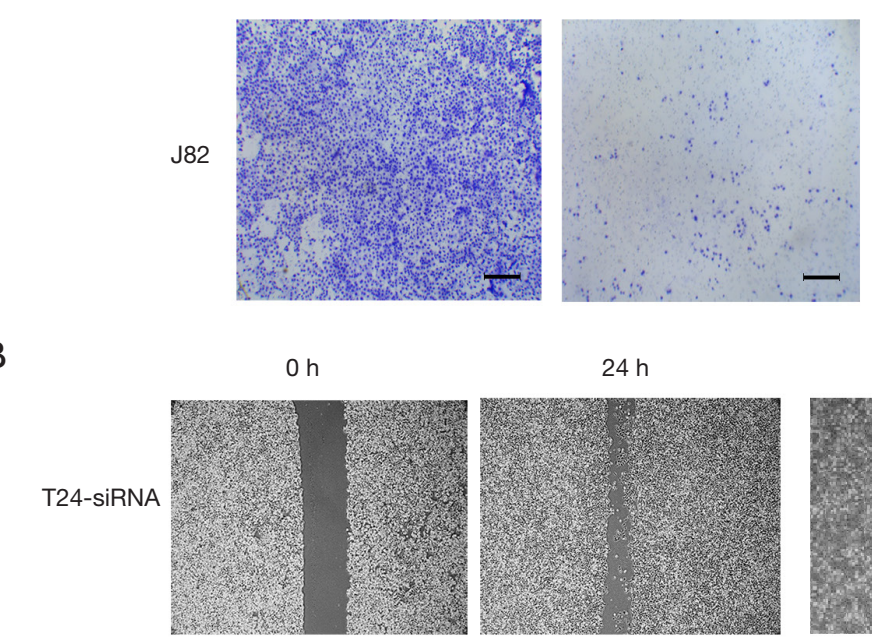

$24 \mathrm{~h}$
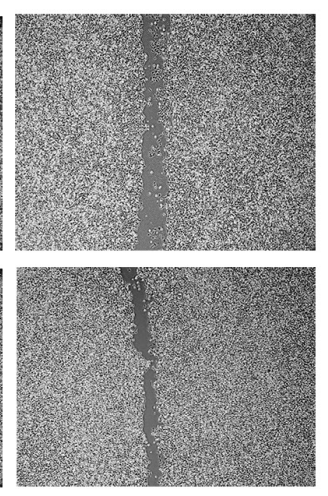

J82

C

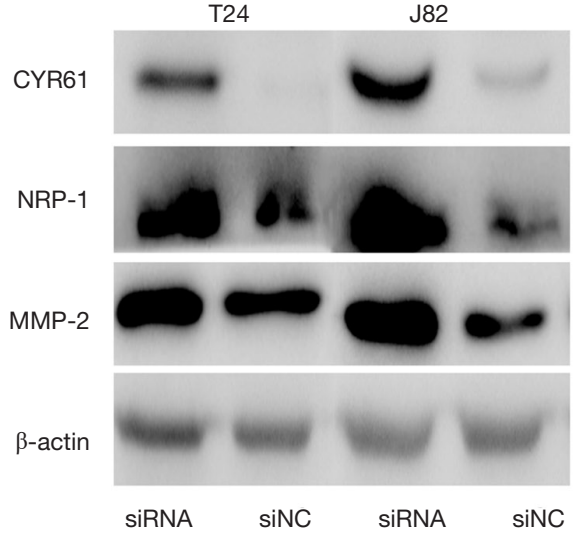

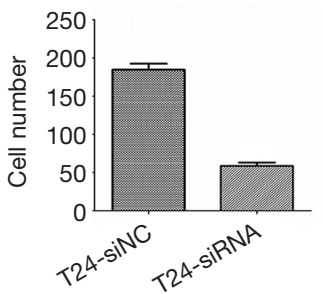

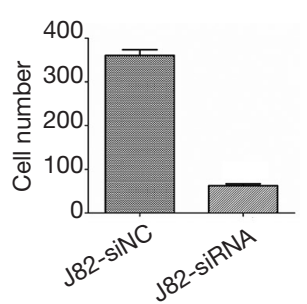

$\mathrm{Oh}$
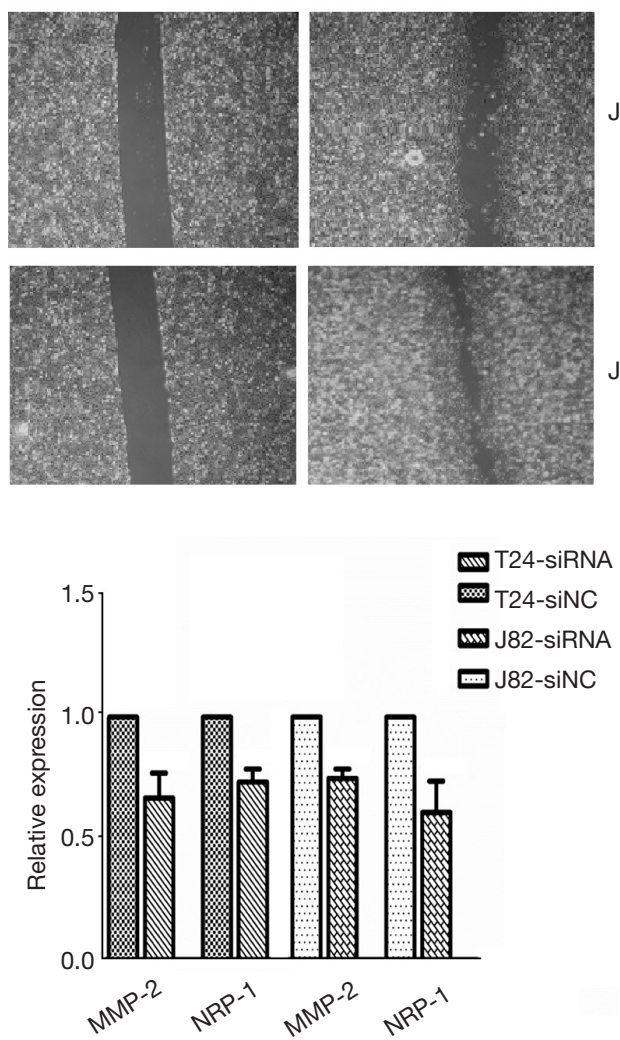

Figure 6 Transiently silenced CYR61 decreased migration and invasion in T24 and J82 cells. CYR61-siNC: interference of negative control group; CYR61-siRNA: silence of CYR61 in T24 and J82 cells. (A) Decreased migration though transwell chamber migration assay $(\mathrm{P}<0.05)$, scale bars: $120 \mu \mathrm{m}$. (B) Wound closure in scratch wounding migration assay $(100 \mathrm{x})$. Photographs were taken at $24 \mathrm{~h}$. (C) Downregulated expression of MMP-2 ( $\mathrm{P}=0.025)$ and NRP-1 ( $\mathrm{P}=0.031)$. CYR61, cysteine-rich angiogenic inducer 61; MMP-2, matrix metalloproteinase-2; NRP-1, neuropilin-1. 
Previous studies have indicated that CYR61 plays a key role in the development of the urogenital system gene. The CYR61 gene is a frequent cause of epispadias or classic bladder exstrophy (38) and could serve as a molecular marker of bladder wall remodeling after outlet obstruction (39). Also, overdistension and cyclic mechanical stretch of bladder smooth muscle cells would significantly increase the mRNA levels of CYR61, which leads to an increase in the expression of VEGF, alpha- $\mathrm{V}$ integrin, and smooth muscle actin $(40,41)$. These phenomena might suggest that CYR61 is a biomarker for MIBC and a key molecule involved in the process of $\mathrm{BC}$ invasion and metastasis.

Therefore, the underlying mechanisms of increased CYR61 in MIBCs were further explored using established BC cell lines. CYR61 has been shown to play a role in the migration and invasion of various cancer (42). However, its role in MIBCs is poorly understood. In our study, overexpressed CYR61 in the 5637 cell line showed no effect on cell invasion, migration, and the expression of MMP-2 and NRP-1. The overexpression of CYR61 in UMUC3 should upregulate cell invasion, migration, and the expression of MMP-2 and NRP-1. Transiently CYR61 silenced T24, J82 cells were deficient in wound healing and invasion, as observed from the scratch and transwell assays. The findings indicate that CYR61 plays a role in the migration and invasion of MIBC cells. The 5637 cell line was derived from NMIBC cells with a low expression of endogenous CYR61. The UMUC3, T24, and J82 cell lines were derived from MIBC cells. The expression of endogenous CYR61 was low in UMUC3 but high in T24 and J82. The findings indicate that the overexpression of CYR61 in vitro can increase cell invasion and migration of MIBCs (J82, T24, and UMUC-3) but cannot increase cell invasion and migration of NMIBCs [5637]. This phenomenon may be related to the expression of wild-type P53 protein in different $\mathrm{BC}$ cells. The P53 gene had doublecopy mutations in J82 cells and single-copy mutations in T24, UMUC-3, and 5637 cells. However, the expression level of wild-type P53 protein in J82, T24, UMUC-3, and 5637 increased. This is similar to other tumors in which mutant P53 has been found to inhibit wild-type P53 and promote tumor cell invasion (43-45).

The present study has some limitations. The majority of patients with NMIBCs recruited in preoperative urine ELISA test had not undergone radical cystectomy; however, the TURBT for NMIBCs confirmed that the tumor had not reached the deep muscle layer, jeopardizing the result. Future studies intend to reevaluate these biomarkers in patients who have exclusively undergone radical cystectomy following preoperative urine ELISA test at a multicenter level. The analyses were not conducted in the same population; however, as transurethral resection of the bladder tumor can result in pseudo-lesions and inaccurate staging (31), all freshlyfrozen tissues included in the present study were from $\mathrm{BC}$ patients who underwent radical or partial cystectomy. The FFPE samples were obtained retrospectively, as the frozen tissue and fresh uric samples could not be acquired. The follow-up period was insufficient to analyze the OS and PFS in both the screening and diagnosis groups. Also, this was a single-center study conducted on small sample size. Only preliminary results from experiments in vitro suggests the role and possible mechanism of CYR61 in MIBC, and further experiments are needed for in-depth research.

\section{Conclusions}

In the present study, we performed systematic screening and validation to identify the biomarker for the preoperative diagnosis of MIBCs. The mRNA and protein expression levels of CYR61 in tissue and urine were significantly higher in patients with MIBCs than those with NMIBCs. Urinary CYR61 had a good accuracy rate for distinguishing MIBCs from NMIBCs, and a combination of other reported biomarkers would achieve good diagnostic efficiency. Therefore, CYR61 can be considered a promising biomarker for the preoperative diagnosis of MIBCs, and a multicentric prospective validation is essential for the further evaluation of CYR61.

\section{Acknowledgments}

Funding: The present study was supported by the Young Foundation of Beijing Union Medical College (grant no. 2012X30), the Doctor Innovation Fund of Beijing Union Medical College (grant No. 2013-1002-49), and the Beijing Hope Run Special Fund of Cancer Foundation of China (grant No. LC2015L12).

\section{Footnote}

Reporting Checklist: The authors have completed the MDAR reporting checklist. Available at http://dx.doi.org/10.21037/ atm-19-4511 
Data Sharing Statement: Available at http://dx.doi. org/10.21037/atm-19-4511

Peer Review File: Available at http://dx.doi.org/10.21037/ atm-19-4511

Conflicts of Interest: All authors have completed the ICMJE uniform disclosure form (available at http://dx.doi. org/10.21037/atm-19-4511). The authors have no conflicts of interest to declare.

Ethical Statement: The authors are accountable for all aspects of the work in ensuring that questions related to the accuracy or integrity of any part of the work are appropriately investigated and resolved. The study was conducted in accordance with the Declaration of Helsinki (as revised in 2013). The study was performed at the Cancer Institute (Hospital), Chinese Academy of Medical Science, Beijing, and was approved by the local ethics committee (No. 12-044/578). All patients provided signed informed consent before enrollment.

Open Access Statement: This is an Open Access article distributed in accordance with the Creative Commons Attribution-NonCommercial-NoDerivs 4.0 International License (CC BY-NC-ND 4.0), which permits the noncommercial replication and distribution of the article with the strict proviso that no changes or edits are made and the original work is properly cited (including links to both the formal publication through the relevant DOI and the license). See: https://creativecommons.org/licenses/by-nc-nd/4.0/.

\section{References}

1. Jemal A, Bray F, Center MM, et al. Global cancer statistics. CA Cancer J Clin 2011;61:69-90.

2. Chen $\mathrm{W}$, Zheng R, Baade PD, et al. Cancer statistics in China, 2015. CA Cancer J Clin 2016;66:115-32.

3. Clark PE, Spiess PE, Agarwal N, et al. NCCN guidelines insights: bladder cancer, version 2.2016. J Natl Compr Canc Netw 2016;14:1213-24.

4. Bellmunt J, Orsola A, Wiegel T, et al. Bladder cancer: ESMO Clinical Practice Guidelines for diagnosis, treatment and follow-up. Ann Oncol 2011;22 Suppl 6:vi45-9.

5. Martignoni G. Discovering smoking-related pathway alterations in urothelial cell carcinoma pathogenesis. Cell
Cycle 2013;12:1483.

6. McLaughlin S, Shephard J, Wallen E, et al. Comparison of the clinical and pathologic staging in patients undergoing radical cystectomy for bladder cancer. Int Braz J Urol 2007;33:25-31; discussion 31-2.

7. Ficarra V, Dalpiaz O, Alrabi N, et al. Correlation between clinical and pathological staging in a series of radical cystectomies for bladder carcinoma. BJU Int 2005;95:786-90.

8. Bayraktar Z, Gurbuz G, Tasci AI, et al. Staging error in the bladder tumor: the correlation between stage of TUR and cystectomy. Int Urol Nephrol 2001;33:627-9.

9. Gaya JM, Matulay J, Badalato GM, et al. The role of preoperative prostatic urethral biopsy in clinical decisionmaking at the time of radical cystectomy. Can J Urol 2014;21:7228-33.

10. Baltaci S, Resorlu B, Yagci C, et al. Computerized tomography for detecting perivesical infiltration and lymph node metastasis in invasive bladder carcinoma. Urol Int 2008;81:399-402.

11. Tekes A, Kamel I, Imam K, et al. Dynamic MRI of bladder cancer: evaluation of staging accuracy. AJR Am J Roentgenol 2005;184:121-7.

12. Huang HY, Shariat SF, Sun TT, et al. Persistent uroplakin expression in advanced urothelial carcinomas: implications in urothelial tumor progression and clinical outcome. Hum Pathol 2007;38:1703-13.

13. Eggers H, Seidel C, Schrader AJ, et al. Serum C-reactive protein: a prognostic factor in metastatic urothelial cancer of the bladder. Med Oncol 2013;30:705.

14. Ramón de Fata F, Ferruelo A, Andrés G, et al. The role of matrix metalloproteinase MMP-9 and TIMP-2 tissue inhibitor of metalloproteinases as serum markers of bladder cancer. Actas Urol Esp 2013;37:480-8.

15. Richter P, Tost M, Franz M, et al. B and C domain containing tenascin-C: urinary markers for invasiveness of urothelial carcinoma of the urinary bladder? J Cancer Res Clin Oncol 2009;135:1351-8.

16. Shafat I, Pode D, Peretz T, et al. Clinical significance of urine heparanase in bladder cancer progression. Neoplasia 2008;10:125-30.

17. Dyrskjøt L, Thykjaer T, Kruhøffer M, et al. Identifying distinct classes of bladder carcinoma using microarrays. Nat Genet 2003;33:90-6.

18. Blaveri E, Simko JP, Korkola JE, et al. Bladder cancer outcome and subtype classification by gene expression. Clin Cancer Res 2005;11:4044-55. 
19. Sanchez-Carbayo M, Socci ND, Lozano J, et al. Defining molecular profiles of poor outcome in patients with invasive bladder cancer using oligonucleotide microarrays. J Clin Oncol 2006;24:778-89.

20. Kim WJ, Kim SK, Jeong P, et al. A four-gene signature predicts disease progression in muscle invasive bladder cancer. Mol Med 2011;17:478-85.

21. Zhang J, Fan Z, Gao Y, et al. Detecting bladder cancer in the Chinese by microsatellite analysis: ethnic and etiologic considerations. J Natl Cancer Inst 2001;93:45-50.

22. Watari H, Xiong Y, Hassan MK, et al. Cyr61, a member of ccn (connective tissue growth factor/cysteine-rich 61/ nephroblastoma overexpressed) family, predicts survival of patients with endometrial cancer of endometrioid subtype. Gynecol Oncol 2009;112:229-34.

23. Jiang WG, Watkins G, Fodstad O, et al. Differential expression of the CCN family members Cyr61, CTGF and Nov in human breast cancer. Endocr Relat Cancer 2004;11:781-91.

24. Vellon L, Menendez JA, Lupu R. AlphaVbeta3 integrin regulates heregulin (HRG)-induced cell proliferation and survival in breast cancer. Oncogene 2005;24:3759-73.

25. Lin MT, Zuon CY, Chang CC, et al. Cyr61 induces gastric cancer cell motility/invasion via activation of the integrin/ nuclear factor-kappaB/cyclooxygenase-2 signaling pathway. Clin Cancer Res 2005;11:5809-20.

26. Lin MT, Kuo IH, Chang CC, et al. Involvement of hypoxia-inducing factor-1 alpha-dependent plasminogen activator inhibitor-1 up-regulation in Cyr61/CCN1induced gastric cancer cell invasion. J Biol Chem 2016;291:27433.

27. Young N, Pearl DK, Van Brocklyn JR. Sphingosine-1phosphate regulates glioblastoma cell invasiveness through the urokinase plasminogen activator system and CCN1/ Cyr61. Mol Cancer Res 2009;7:23-32.

28. Zhang L, Xiao H, Zhou H, et al. Development of transcriptomic biomarker signature in human saliva to detect lung cancer. Cell Mol Life Sci 2012;69:3341-50.

29. Kang CJ, Chen YJ, Liao CT, et al. Transcriptome profiling and network pathway analysis of genes associated with invasive phenotype in oral cancer. Cancer Lett 2009;284:131-40.

30. Tanaka F, Rizqiawan A, Higashikawa K, et al. Snail promotes Cyr61 secretion to prime collective cell migration and form invasive tumor nests in squamous cell carcinoma. Cancer Lett 2013;329:243-52.

31. Divrik RT, Sahin AF, Yildirim U, et al. Impact of routine second transurethral resection on the long-term outcome of patients with newly diagnosed p'T1 urothelial carcinoma with respect to recurrence, progression rate, and diseasespecific survival: a prospective randomised clinical trial. Eur Urol 2010;58:185-90.

32. Marín-Aguilera M, Mengual L, Burset M, et al. Molecular lymph node staging in bladder urothelial carcinoma: impact on survival. Eur Urol 2008;54:1363-72.

33. Goodison S, Chang M, Dai Y, et al. A multi-analyte assay for the non-invasive detection of bladder cancer. PLoS One 2012;7:e47469.

34. Lin $\mathrm{Y}, \mathrm{Xu} \mathrm{T}$, Tian $\mathrm{G}$, et al. Cysteine-rich, angiogenic inducer, 61 expression in patients with ovarian epithelial carcinoma. J Int Med Res 2014;42:300-6.

35. Lin J, Li N, Chen H, et al. Serum Cyr61 is associated with clinical disease activity and inflammation in patients with systemic lupus erythematosus. Medicine (Baltimore) 2015;94:e834.

36. Klingenberg R, Aghlmandi S, Liebetrau C, et al. Cysteinerich angiogenic inducer 61 (Cyr61): a novel soluble biomarker of acute myocardial injury improves risk stratification after acute coronary syndromes. Eur Heart J 2017;38:3493-502.

37. Schiffer E, Vlahou A, Petrolekas A, et al. Prediction of muscle-invasive bladder cancer using urinary proteomics. Clin Cancer Res 2009;15:4935-43.

38. Draaken M, Proske J, Schramm C, et al. Embryonic expression of the cysteine rich protein 61 (CYR61) gene: A candidate for the development of human epispadias. Birth Defects Res A Clin Mol Teratol 2010;88:546-50.

39. Chaqour B, Whitbeck C, Han JS, et al. Cyr61 and CTGF are molecular markers of bladder wall remodeling after outlet obstruction. Am J Physiol Endocrinol Metab 2002;283:E765-74.

40. Zhou D, Herrick DJ, Rosenbloom J, et al. Cyr61 mediates the expression of VEGF, alphav-integrin, and alpha-actin genes through cytoskeletally based mechanotransduction mechanisms in bladder smooth muscle cells. J Appl Physiol (1985) 2005;98:2344-54.

41. Tamura I, Rosenbloom J, Macarak E, et al. Regulation of Cyr61 gene expression by mechanical stretch through multiple signaling pathways. Am J Physiol Cell Physiol 2001;281:C1524-32.

42. Liang CC, Park AY, Guan JL. In vitro scratch assay: a convenient and inexpensive method for analysis of cell migration in vitro. Nat Protoc 2007;2:329-33.

43. Cooper MJ, Haluschak JJ, Johnson D, et al. p53 mutations in bladder carcinoma cell lines. Oncol Res 
1994;6:569-79.

44. Tang Y, Simoneau AR, Xie J, et al. Effects of the kava chalcone flavokawain A differ in bladder cancer cells with wild-type versus mutant p53. Cancer Prev Res (Phila) 2008;1:439-51.

Cite this article as: Chen $\mathrm{H}$, Liu Y, Cao C, Xi H, Chen W, Zheng W, Dong X, Zheng S, Li L, Ma J, Gao Y, Shou J. CYR61 as a potential biomarker for the preoperative identification of muscle-invasive bladder cancers. Ann Transl Med 2021;9(9):761. doi: $10.21037 /$ atm-19-4511
45. Ali A, Shah AS, Ahmad A. Gain-of-function of mutant p53: mutant p53 enhances cancer progression by inhibiting KLF17 expression in invasive breast carcinoma cells. Cancer Lett 2014;354:87-96. 


\section{Supplementary}

Table S1 Demographics and tumor characteristics of patients in screening set and validation set

\begin{tabular}{|c|c|c|c|c|c|c|}
\hline Characteristics & \multicolumn{3}{|c|}{ Screening set $(n=30), n(\%)$} & \multicolumn{3}{|c|}{ Validation set $(n=54), n(\%)$} \\
\hline Age, years & & & 0.815 & & & 0.561 \\
\hline Mean \pm SD & $63.27 \pm 13.03$ & $64.87 \pm 10.89$ & & $63.03 \pm 10.45$ & $61.18 \pm 12.27$ & \\
\hline Male & $9(56.3)$ & $9(64.3)$ & & $28(87.5)$ & $19(86.3)$ & \\
\hline Female & $7(43.7)$ & $5(35.7)$ & & $4(12.5)$ & $3(13.7)$ & \\
\hline Drink & & & 0.134 & & & 0.583 \\
\hline Yes & $4(25.0)$ & $6(42.9)$ & & $11(34.4)$ & $10(45.5)$ & \\
\hline No & $12(75.0)$ & $8(57.1)$ & & $21(65.6)$ & $12(54.5)$ & \\
\hline Tumor grade & & & 0.732 & & & 0.071 \\
\hline Low & $7(43.8)$ & $7(50.0)$ & & $15(46.9)$ & $5(22.7)$ & \\
\hline High & 9 (56.3) & $7(50.0)$ & & $17(53.1)$ & $17(77.3)$ & \\
\hline Multifocality & & & 0.654 & & & 0.165 \\
\hline$>1$ & 9 (56.3) & 9 (64.3) & & $22(68.8)$ & $11(50.0)$ & \\
\hline
\end{tabular}

SD, standard deviation. 
A

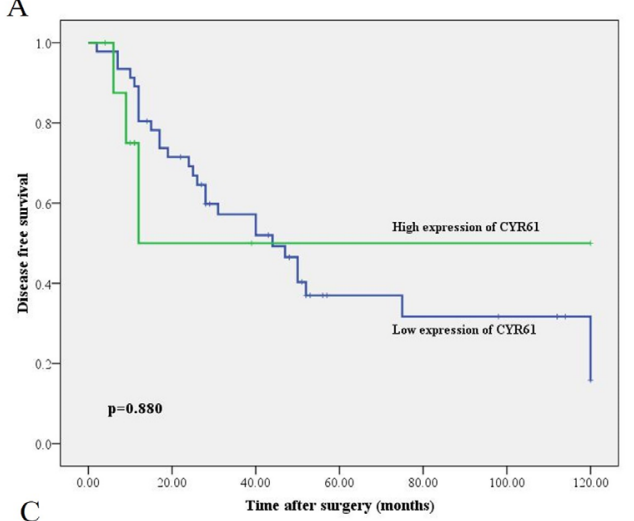

C

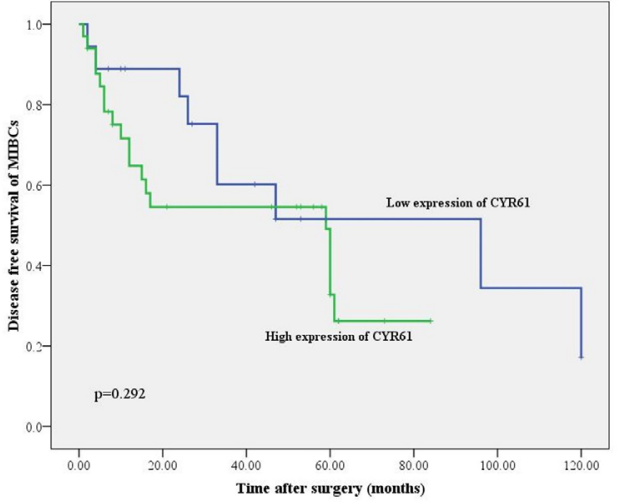

B
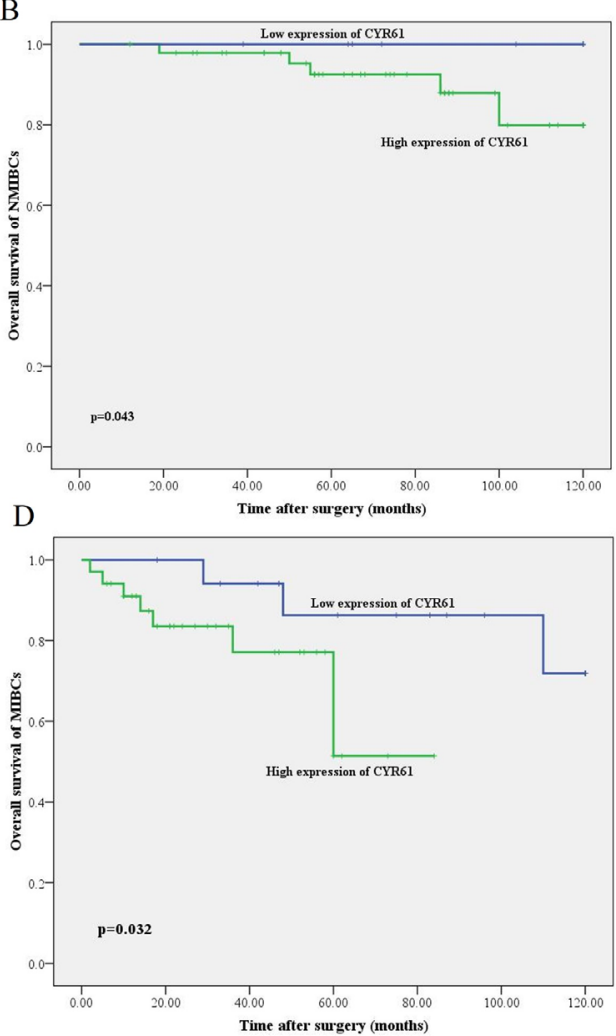

Figure S1 Kaplan-Meier analysis of CYR61 expression in NMIBCs (n=56) and MIBC (n=52). (A) Relationship of CYR61 expression and NMIBCs patients' disease-free survival: low expression, $\mathrm{n}=46$; high expression, $\mathrm{n}=10(\mathrm{P}=0.880, \log -$ rank $=0.023)$. (B) OS curves of NMIBCs by CYR61 expression ( $\mathrm{P}=0.043$, log-rank =5.235). (C) Relationship of CYR61 expression and MIBCs patients' disease-free survival: low expression, $\mathrm{n}=28$; high expression, $\mathrm{n}=10(\mathrm{P}=0.292$, log-rank =1.111). (D) OS curves of NMIBCs by CYR61 expression (P=0.032, log-rank =7.213). CYR61, cysteine-rich angiogenic inducer 61; NMIBC, non-muscle-invasive bladder cancer; MIBC, muscle-invasive bladder cancer; OS, overall survival.

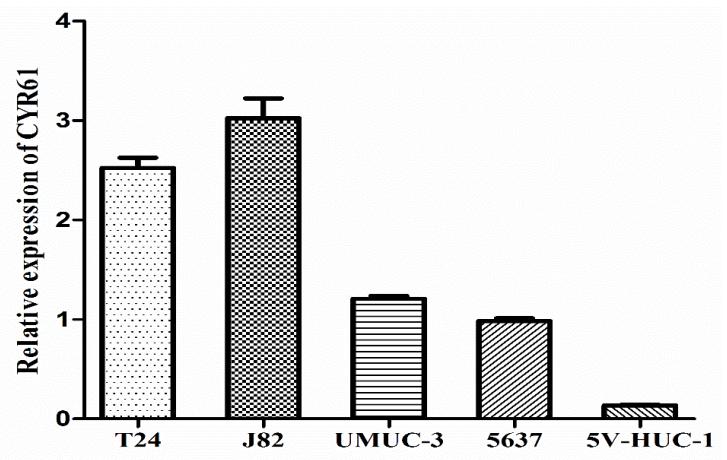

CYR61

$\beta$-Actin

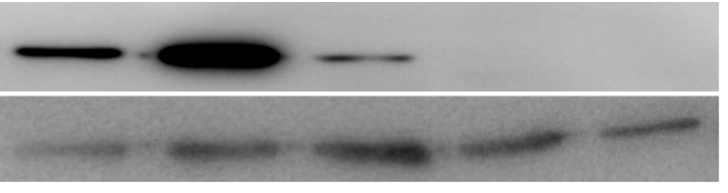

T24 J82 UM-UC-3 $5637 \quad$ SV-HU-1

Figure S2 Endogenous levels of CYR61 in different bladder carcinoma cells. CYR61, cysteine-rich angiogenic inducer 61. 

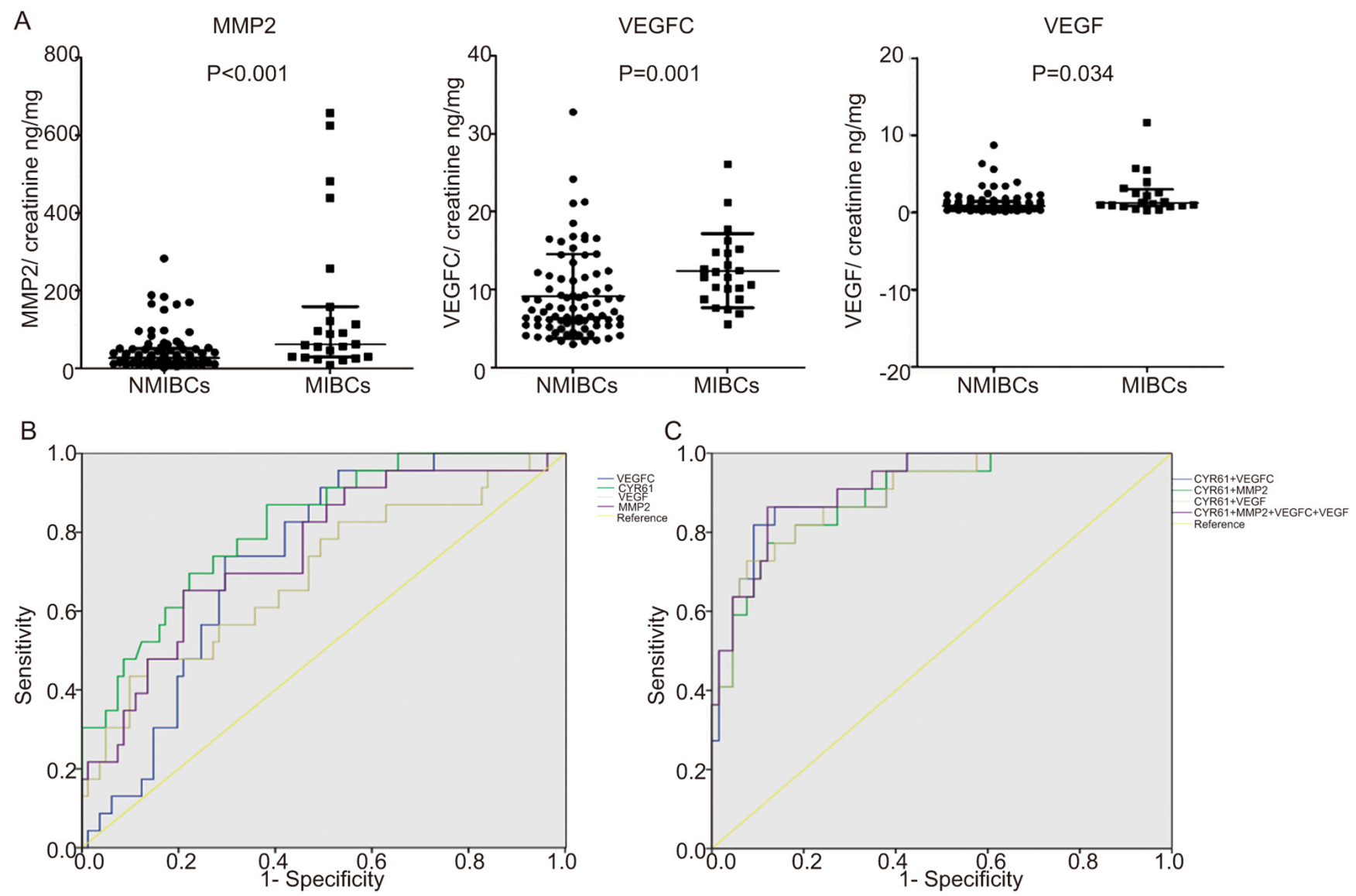

Figure S3 Urine concentration and ROC curves of MMP-2, VEGFC, and VEGF in NMIBCs and MIBCs. (A) Urine concentration of MMP-2, VEGFC, and VEGF in NMIBCs and MIBCs. (B) ROC curves were plotted to compare the diagnostic performance of CYR61, MMP-2, VEGFC, and VEGF individually. (C) ROC curves were plotted to compare the diagnostic performance of CYR61, MMP-2, VEGFC, and VEGF together. ROC, receiver-operating characteristic; MMP-2, matrix metalloproteinase-2; NMIBC, non-muscle-invasive bladder cancer; MIBC, muscle-invasive bladder cancer; CYR61, cysteine-rich angiogenic inducer 61.

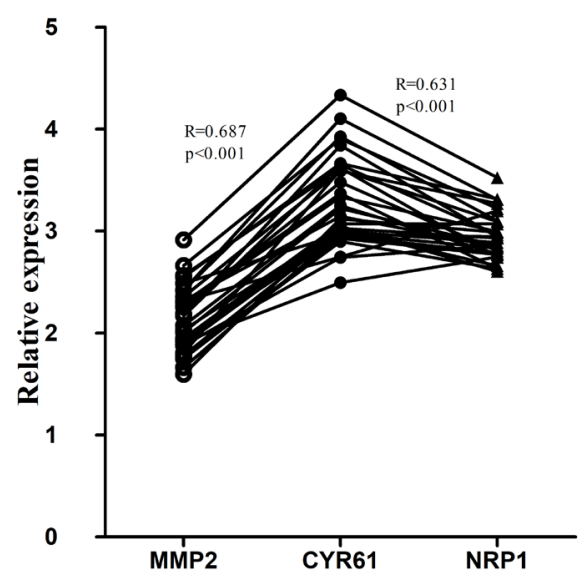

Figure S4 The correlation between expression of CYR61 and MMP-2/NRP-1 in the gene expression profiling of 30 human bladder tumors. CYR61, cysteine-rich angiogenic inducer 61; MMP-2, matrix metalloproteinase-2; NRP-1, neuropilin-1. 
Table S2 Diagnostic performance of CYR61, MMP-2, VEGFC, and VEGF to distinguish MIBC from NMIBC individual

\begin{tabular}{lccccc}
\hline Biomarker & AUC & P value & Cutoff value $(\mathrm{ng} / \mathrm{mg})$ & Sensitivity & Specificity \\
\hline CYR61 & 0.883 & $<0.001$ & 4.596 & 0.727 & 0.860 \\
MMP-2 & 0.806 & $<0.001$ & 54.695 & 0.682 & 0.864 \\
VEGFC & 0.796 & $<0.001$ & 9.939 & 0.727 & 0.788 \\
VEGF & 0.736 & 0.001 & 0.790 & 0.818 & 0.561 \\
\hline
\end{tabular}

CYR61, cysteine-rich angiogenic inducer 61; MMP-2, matrix metalloproteinase-2; MIBC, muscle-invasive bladder cancer; NMIBC, nonmuscle-invasive bladder cancer; AUC, area under the curve. 